\title{
O SEVOFLUORANO EM PSITACÍDEOS (Amazonas aestiva). DETERMINAÇÃO DA DOSE MÍNIMA (D. A. M) PARA PRODUÇÃO DE ANESTESIA GERAL ${ }^{1}$
}

\author{
SEVOFLURANE IN PSITACINES (Amazonas aestiva). DETERMINATION \\ OF MINIMAL ANESTHETIC CONCENTRATION \\ TO PRODUCE GENERAL ANESTHESIA
}

\author{
Alexandra Alves Nicolau ${ }^{2}$ Denise Tabbachi Fantoni ${ }^{3}$ \\ José Otávio Auler ${ }^{4}$ Aline Ambrósio 5
}

RESUMO

Foram utilizados 10 psitacídeos da espécie Amazonas aestiva (papagaio-verdadeiro). Após contenção física, a anestesia foi induzida com o auxílio de uma máscara facial conectada ao sistema de Maplesson D (BARAKA), utilizando sevofluorano a $6 \mathrm{~V} \%$ e fluxo diluente de $\mathrm{O}_{2}$ de $1,5 \mathrm{l} / \mathrm{min}$ de oxigênio. A determinação da Dose Anestésica Mínima (DAM) foi estabelecida de maneira similar àquela proposta por LUDDERS et al. (1990). A DAM obtida neste experimento 3,44V\%, que eqüivale a concentração alveolar mínima de mamíferos mostrouse maior nas aves analisadas quando comparada a obtida nos mamíferos. $O$ anestésico mostrou-se bastante seguro para a espécie, não alterando de forma deletéria as variáveis fisiológicas analisadas.

Palavras-chave: anestesia inalatória; aves; anestésicos inalatórios; CAM.

\section{SUMMARY}

Ten adult psittacines (Amazonas aestiva)were used After appropriate immobilization the anesthetic induction was accomplished with facial mask connect to modified circuit of Maggil using $6 \mathrm{~V} \%$ of Sevofluorano with oxygen flow rate at 1.5/l. The Minimal Anesthetic Concentration was determined in a manner similar to that proposed by LUDDERS et al.(1990). The MAC obtained was $3.44 \mathrm{~V} \%$, higher than MAC. proposed to mammals. Sevoflurane can be used safely in avian species once it did not alter significantly all physiological parameters analyzed.

Key words: inhalation anesthesia; avian; inhalation anesthetics; CAM.

\section{INTRODUÇÃO}

Nos últimos anos, não só pelo aumento de interesse na conservação da vida selvagem, mas também pelo crescente hábito de manter aves como animais de companhia, cresceu a demanda para contenção e/ou anestesia de aves selvagens ou semiselvagens (HALL \& CLARKE, 1987; CURRO et al., 1994). Assim, o médico veterinário enfrenta a necessidade de executar a contenção química e/ou anestesia nas espécies aviárias (mais de 850 espécies) dos mais diferentes tamanhos, comportamentos e hábitos alimentares. Por isso, torna-se impossível estabelecer uma dose aplicável dos diferentes agentes imobilizantes para esta

\footnotetext{
${ }^{1}$ Parte da tese de doutorado, apresentada pela primeira autora para obtenção do grau de Doutor em Patologia pela Universidade de São Paulo (USP), São Paulo, SP.

${ }^{2}$ Médico Veterinário, Mestre. Av. Fernando Ferrari, 255, 09760-110, Ferrazopoli SBC, SP. E-mail: alenicolau@hotmail.com. Autor para correspondência.

${ }^{3}$ Médico Veterinário, Doutor, Professor Livre-Docente, Departamento de Cirurgia Veterinária da USP.

${ }^{4}$ Médico, Doutor, Professor Titular, Departamento de Anestesiologia , Faculdade de Medicina da USP.

${ }^{5}$ Médico Veterinário, Mestre Doutorando do Departamento de Cirurgia Veterinária da USP.
} 
diversidade de aves (BOEVER \& WRIGHT, 1975). Acrescentam-se, ainda, diferenças de ordem anatômica, fisiológica, comportamental e metabólica existentes entre as aves e os mamíferos, que não permitem a extrapolação de doses entre eles (HALL \& CLARKE, 1987; SCHMITT et al., 1998).

Os anestésicos inalatórios são os mais escolhidos para a anestesia de aves por possuírem alto índice de segurança, permitirem indução e recuperação rápida da anestesia, serem pouco metabolizados, e facilitarem alteração dos planos anestésicos, sobretudo porque o tempo de anestesia é ilimitado. Porém, têm o inconveniente de requererem equipamento adequado, nem sempre disponível (LUMB \& JONES, 1984).

Dentre os agentes inalatórios mais empregados em aves, têm-se o óxido nitroso, o halotano e o isoflurano. Dentre estes, ROSSKOPF \& WOERPEL (1996) ressaltam que a maioria dos clínicos aviários americanos considera o isoflurano como o agente anestésico mais seguro, sendo que os demais deveriam ser empregados apenas em situações emergenciais, quando o isoflurano não estiver disponível.

Recentemente, em meados de 1997 , introduziu-se no Brasil um novo anestésico inalatório halogenado, o sevofluorano. Esse não é inflamável, possui odor agradável, estruturalmente similar ao isoflurano e ao enflurano. $\mathrm{O}$ maior produto de sua hidrólise é o hexafluoroisopropanol, que é excretado na urina como conjugado glicurônico (HIKASA et al., 1996). Possui um coeficiente de partição sangue/gás bastante baixo $(0,69)$, que é similar ao óxido nitroso, o que acarreta rápida indução e recuperação da anestesia quando comparado ao isoflurano e o halotano. NOCITI (1998) demostrou que a indução anestésica em pacientes pediátricos, produzida com o emprego isolado do sevofluorano, foi de excelente qualidade, podendo ser obtida com sucesso em 3,3 $\pm 1,2$ minutos. Essa excelência pode ser atribuída, em parte, pela baixa solubilidade sanguiínea e, ainda, ao odor agradável desse agente (NOCITI, 1998).

Em relação à sua ação no sistema cardiovascular, sabe-se que o sevofluorano produz menores alterações na freqüência cardíaca, na pressão sangüínea e no débito cardíaco quando comparado ao isoflurano, com mínimo risco de arritmias ventriculares espontâneas (FRINK et al., 1992). Por conservar as funções cardiovasculares, é indicado para pacientes pediátricos, geriátricos ou aqueles que apresentam maior comprometimento das funções orgânicas (MÂNICA, 1997). O ponto de ebulição e a pressão de vapor são muito semelhantes aos de outros anestésicos, fato esse que permite a utilização desse agente em vaporizadores calibrados já disponíveis no Brasil.

A concentração alveolar mínima (CAM), que se refere à concentração na qual $50 \%$ dos indivíduos não respondem a estímulos dolorosos, serve como medida de potência de um anestésico. Embora tenha sido usada para descrever essa variável em aves, a sigla CAM vem sendo substituída pelo termo Dose Anestésica Mínima, uma vez que, nas aves, o sistema respiratório difere daquele dos mamíferos (LUDDERS \& MATTHEWS, 1996). Nas aves, este sistema não é composto de um pulmão alveolar, e sim de dois componentes distintos funcionalmente: um componente para ventilação (vias condutoras de ar, sacos aéreos, esqueleto torácico, músculos da respiração) e componentes para troca de gases denominados parabrônquios, que são capilares aéreos que se anastomosam com capilares sangüíneos (LUDDERS \& MATTHEWS, 1996).

Existem poucas referências disponíveis na literatura sobre o emprego do sevofluorano nas espécies aviárias e a determinação de sua DAM. Por suas características físico-químicas, espera-se que esse agente produza rápida indução e recuperação da anestesia, baixa metabolização e estabilidade cardiovascular. Assim, neste trabalho, observaramse os efeitos deste anestésico administrado isoladamente para produção de contenção química e/ou anestesia e a determinação da DAM do sevofluorano para psitacídeos.

\section{MATERIAIS E MÉTODOS}

Foram utilizados 10 psitacídeos da espécie Amazonas aestiva (papagaio-verdadeiro) provenientes de setores extras do Parque Ecológico do Tietê, localizado na cidade de São Paulo - SP . As aves foram retiradas do recinto moradia e alocadas em gaiolas de arame, 18 horas antes do experimento, para que houvesse um período de adaptação. O tempo de jejum hídrico e sólido foi estabelecido segundo RITCHIE et al. (1997) e correspondeu a um período de 4 horas.

\section{Indução e manutenção da anestesia}

Após adequada contenção física, a anestesia foi induzida com o auxílio de uma máscara neonatal adaptada a uma luva de latex, para evitar vazamentos, com o sevofluorano vaporizado em $100 \%$ de oxigênio. A concentração inicial de sevofluorano foi aquela necessária para promover anestesia cirúrgica (perda do reflexo interdigital, laringo-traqueal e ausência de reflexo palpebral) nas aves e correspondeu a $6 \mathrm{~V} \%$. $\mathrm{O}$ reflexo interdigital 
foi avaliado com o auxílio de uma pinça no espaço interdigital da ave. O reflexo laringo-traqueal foi avaliado através do procedimento de intubação e o palpebral foi avaliado tocando-se gentilmente o canto interno do olho das aves. O circuito empregado foi o de Baraka, ou seja, sem reinalação, com fluxo de oxigênio calculado (1,5 $\ell / \mathrm{min})$ para que se obtivesse valor de dióxido de carbono no ar inspirado inferior a $2 \mathrm{mmHg}$. Empregou-se vaporizador próprio para sevofluorano. A DAM foi estabelecida de maneira similar àquela proposta por LUDDERS et al. (1990).

Uma concentração inicial equivalente a $6 \mathrm{~V} \%$ de sevofluorano foi administrada para cada ave. Esperaram-se 7 minutos para o estabelecimento do equilíbrio entre a concentração parabronquial e cerebral do anestésico. Logo após, avaliou-se o reflexo interdigital. Para tanto, a ave foi estimulada com auxílio de uma pinça hemostática, a qual foi fechada completamente, utilizando-se seu comprimento total, no espaço interdigital da ave, durante um minuto. Quando a resposta (levantar de cabeça, bater de asas ou flexionar os joelhos) a esse estímulo não ocorreu, a concentração do sevofluorano foi reduzida em $20 \%$ da inicial. Então, aguardou-se mais um minuto para o restabelecimento do equilíbrio quando, então, o estímulo foi repetido. Esse procedimento foi realizado até que a ave respondesse ao estímulo doloroso. Ocorrido isto, a concentração de sevofluorano foi aumentada em $10 \%$ e, após um minuto de equilíbrio, o estímulo foi repetido. $\mathrm{O}$ valor da concentração de sevofluorano no ar expirado que previne movimentos foi determinada como a sua DAM.

Após a administração do sevofluorano, a qualidade da indução anestésica foi avaliada pela facilidade da intubação orotraqueal e presença (+) ou ausência (-) de excitação, através de movimentos de pedalagem, bater de asas e vocalizações. A frequiência cardíaca, na unidade de tempo minuto, foi obtida com o auxílio de um estetoscópio, contabilizando-se, através da média de duas contagens, em um espaço de 15 segundos cada, sendo, posteriormente, multiplicado para obter o valor correspondente a um minuto. A freqüência respiratória foi obtida através do analisador de gases anestésicos e foi registrada no decorrer de um minuto. A coleta de sangue para hemogasometria foi realizada através da punção da artéria braquial. $O$ volume coletado foi de $0,2 \mathrm{~m} \ell$ em seringa descartável heparinizada (TERZI, 1992), cuja agulha foi vedada por tampa de borracha para evitar, assim, contato do sangue com o ar ambiente. Em seguida, a amostra foi acondicionada em recipiente isotérmico contendo gelo e água, para posterior análise em analisador de $\mathrm{pH}$ e gases sangüíneos. Essas amostras foram analisadas no final de cada experimento avaliando-se o $\mathrm{pH}$ arterial, pressão parcial do oxigênio (pO2), pressão parcial de dióxido de carbono (pCO2), bicarbonato plasmático arterial $\left(\mathrm{HCO}^{-}\right)$e saturação da oxihemoglobina arterial. A temperatura retal foi mensurada através do uso de termômetro clínico de mercúrio introduzido na cloaca do animal, até atingir o valor máximo estável, sendo seu valor registrado em graus Celsius $\left({ }^{\circ} \mathrm{C}\right)$. Avaliou-se também a pressão arterial sistólica mediante o emprego de "doppler ultrassonic" sendo o manguito colocado na região da artéria tibial medial da ave.

\section{Recuperação anestésica}

A partir da interrupção da administração do anestésico, as aves foram avaliadas na sua recuperação anestésica quanto ao tempo decorrido para movimentação do pescoço e imediata extubação; tempo decorrido para entrar em decúbito esternal; tempo para adquirir posição bipedal e, ainda, o tempo para o total restabelecimento da atividade normal.

Considerou-se, para tal avaliação, o fato de o animal apresentar-se em estação, caminhando normalmente e apto para se locomover pela gaiola. A qualidade da recuperação anestésica foi avaliada pela presença ou ausência de ataxia e excitação (movimentos exacerbados de asas).

\section{RESULTADOS E DISCUSSÃO}

As tabelas de 1 a 3 demostram os resultados obtidos. A anestesia de aves, em grande parte, constitui um desafio, tendo-se em vista o pequeno número de estudos na área aliado à grande diversidade de espécies encontradas na clínica diária. Esses fatos dificultam sobremaneira o

Tabela 1 - Valores da DAM, freqüência cardíaca, freqüência respiratória, pressão arterial sistólica e temperatura retal de papagaios anestesiados com $\mathrm{Se}$ vofluorano. Estão apresentadas as médias e desvio-padrões. $(\mathrm{M}=$ média; $\mathrm{DP}=$ desvio-padrão; DAM = dose anestésica mínima; $\mathrm{FR}=$ frequência respiratória; $\mathrm{FC}=$ freqüência cardíaca; $\mathrm{PAM}=$ pressão arterial sistólica; $\mathrm{TC}=$ temperatura retal.

\begin{tabular}{lccccc}
\hline Ave & $\begin{array}{c}\text { DAM } \\
(\%)\end{array}$ & $\begin{array}{c}\text { FR } \\
(\mathrm{bpm})\end{array}$ & $\begin{array}{c}\text { FC } \\
(\mathrm{mpm})\end{array}$ & $\begin{array}{c}\text { PAS } \\
(\mathrm{mmmHg})\end{array}$ & $\begin{array}{c}\text { TC } \\
\left({ }^{\circ} \mathrm{C}\right)\end{array}$ \\
\hline $\mathrm{M} \pm \mathrm{DP}$ & $3,44 \pm 0,25$ & $13 \pm 4,57$ & $283,2 \pm 35,36$ & $172,2 \pm 25,46$ & $38,75 \pm 1,45$ \\
\hline
\end{tabular}

Ciência Rural, v. 32, n. 5, 2002. 
Tabela 2 - Médias e desvios-padrão dos valores de sevofluorano inspirado (\%) e expirado, pH e gases sangüíneos de papagaios anestesiados com sevofluorano.

\begin{tabular}{lcccccc}
\hline Ave & $\begin{array}{c}\text { \% sevo } \\
\text { insp }\end{array}$ & $\begin{array}{c}\% \text { sevo } \\
\exp \end{array}$ & $\mathrm{pH}$ & $\mathrm{Sat} \mathrm{O}_{2}$ & $\mathrm{PCO}_{2}$ & $\mathrm{PO}_{2}$ \\
\hline $\mathrm{M} \pm \mathrm{DP}$ & $3,61 \pm 0,29$ & $3,44 \pm 0,25$ & $7,29 \pm 0,04$ & $98,9 \pm 1,96$ & $55,87 \pm 8,28$ & $255,8 \pm 99,98$
\end{tabular}

emprego dos anestésicos injetáveis, pois, como é de se esperar, as doses deveriam ser distintas para as diferentes espécies. Entretanto, encontra-se na literatura certa generalização no que alude às doses dos fármacos injetáveis, estudos enfocando apenas as espécies mais comuns e pesquisas feitas com emprego de um pequeno número de animais. Assim, o emprego de anestesia inalatória é interessante nas aves por diferentes motivos: facilidade de se alterar os planos de anestesia, tempo não ser fator limitante, ausência de efeitos hepáticos e renais em se tratando dos anestésicos pouco metabolizados, como a sevofluorano ou isoflurano. Aliada a essas vantagens, está a possibilidade de se empregarem os agentes inalatórios mesmo em espécies as quais não se conhece exatamente o valor da CAM, uma vez que, tomadas as devidas precauções, há possibilidade de promover a anestesia gradativamente e controlar os planos mediante os reflexos protetores, parâmetros cardiovasculares e respiratórios. Entretanto, esta prática pode não ser desejável, sobretudo quando se leva em conta a importância de se preservarem algumas espécies e o valor sentimental que os animais, quando de companhia, possam representar.

Os anestésicos inalatórios mais modernos, por suas características físico-químicas como baixos coeficientes de partição óleo-gás e sangue gás, como os do isoflurano, sevofluorano e desflurano, fazem com que a indução da anestesia seja extremamente rápida, o que muitas vezes exclui a necessidade do uso de outros agentes (OLKOWSKY \& CALSSEN, 1998; ALTMAN; 1998 MUTOH et al., 1997). É fato que a contenção inicial para a administração do anestésico poderia ser um fator deletério, mas, devido ao fato de esses fármacos causarem indução extremamente rápida, isso passa a não ser mais um problema. De qualquer forma, o emprego do anestésico injetável também obriga o mesmo tipo de contenção.
No presente estudo, o sevofluorano foi escolhido para ser avaliado uma vez que no, homem e nos mamíferos em geral, relata-se que o isoflurano, por possuir odor pungente e promover irritação de mucosa, não seria o agente mais indicado para a indução da anestesia sem prévia administração de agente intravenoso (DOI \& IKEDA, 1993) No entanto, com o advento de sevofluorano, esse agente passou a ser o fármaco de eleição para a indução na máscara por não acarretar esse problema (BRAGA et al., 1998; LAURETTI et al., 1998).

O sevofluorano promove pouca depressão cardiovascular quando comparado ao halotano (MUTOH et al., 1997). A frequiência cardíaca aumenta e a pressão arterial pode diminuir de forma dosedependente. No presente estudo, verificou-se que a frequiência cardíaca se manteve entre os valores normalmente aceitos para animais com peso ao redor de quatrocentos gramas sem anestesia (RITCHIE $\boldsymbol{e t a l}$., 1997). Quando no sistema respiratório, os anestésicos inalatórios deprimem a freqüência respiratória de forma significativa durante respiração espontânea, ocasionando aumento da $\mathrm{PaCO}_{2}$ e diminuição do volume minuto (PAULIN, 1986). As respostas ventilatórias à hipercapnia e a hipóxia encontram-se diminuídas em decorrência da depressão da atividade dos quimiorreceptores. O isoflurano, dentre os anestésicos halogenados, é considerado o mais depressor do sistema respiratório (PAULIN, 1986; STEFFEY, 1996). A freqüência respiratória foi inferior aos valores basais para a ave com $400 \mathrm{~g}$ de peso corpóreo. Essa depressão já era esperada, uma vez que todos os anestésicos inalatórios causam este efeito por deprimirem o centro respiratório (STEFFEY, 1996). O que vem corroborar esses achados são os valores de $\mathrm{pH}$ e $\mathrm{PaCO}_{2}$. Embora não haja na literatura compulsada os valores basais para psitacídeos, comparando-os com os de outras espécies, verifica-se que os valores encontrados estão abaixo do normal, demonstrando claramente a depressão respiratória. LUDDERS et al., (1990) verificaram valores de $\mathrm{pH}$ em torno de 7,31 em patos submetidos a 1,5 DAM de isoflurano. No entanto, em galinhas anestesiadas com o sevofluorano, GUIMARÃES et $\boldsymbol{a l}$. (2000) demostraram um valor de pH de 7,44. Já em relação à $\mathrm{PaCO}_{2}$, os valores se

Tabela 3 - Médias e desvios-padrão do tempo de indução, recuperação anestésica de papagaios anestesiados com sevofluorano.

\begin{tabular}{|c|c|c|c|c|c|}
\hline Ave & Tempo.de indução (seg) & Mov.de pescoço (seg) & Decúbito esternal (seg) & Posição bipedal (seg) & Recuperação. Total (seg) \\
\hline Média $\pm D P$ & $66,4 \pm 18,47$ & $83,1 \pm 15,01$ & $135,2 \pm 30,31$ & $228,5 \pm 35,87$ & $256,7 \pm 54,54$ \\
\hline
\end{tabular}

Ciência Rural, v. 32, n. 5, 2002. 
equivalem àqueles verificados por LUDDERS $\boldsymbol{e t}$ al . (1990), uma vez que esses autores encontraram valores da ordem de $56 \mathrm{mmHg}$ e no presente estudo foram encontrados 55,87 $\mathrm{mmHg}$. No que alude aos valores de freqüência respiratória, comparativamente aos valores verificados nos patos de LUDDERS et al. (1990), verificou-se nos papagaios melhor manutenção deste parâmetro, fato esse relacionado, provavelmente, à menor depressão promovida pelo sevofluorano, o que, no entanto, é impossível inferir com segurança, uma vez que não está definido o valor basal desta espécie. A $\mathrm{PaO}_{2}$, no presente estudo, foi em torno de $255 \mathrm{mmHg}$, enquanto que LUDDERS et al. (1990) verificaram valores de $358 \mathrm{mmHg}$. Também dizer que os patos, quando submetidos à anestesia com isoflurano, deprimiram menos que os papagaios com sevofluorano não é pertinente, pois não foi relatada a fração inspirada de oxigênio nos momentos da mensuração das amostras nos patos. No presente estudo, o valor da $\mathrm{FiO}_{2}$ era $60 \%$. Considerando-se a relação $\mathrm{PaO}_{2} / \mathrm{FiO}_{2}$, podese afirmar que, no que tange à oxigenação, os papagaios estavam sendo adequadamente oxigenados, levando-se em conta o fato de verificar-se valor acima de $200 \mathrm{mmHg}$ para esse parâmetro.

GOELZ et al. (1990) colocam que o tempo de indução da anestesia com isoflurano em patos foide $4.2 \pm 1.0$ minutos. Já para KORBEL (1998), que avaliou o sevofluorano em pombos, o tempo foi de 1 minuto e $34 \pm 9$ segundos. No estudo em tela, obtevese 1 minuto e $6 \pm 18$ segundos como tempo de indução, corroborando com os achados de KORBEL (1998) e com as características físico-químicas deste agente. A DAM obtida neste experimento, que equivale à concentração alveolar mínima de mamíferos, mostrouse maior nas aves analisadas quando comparadas as DAM obtidas nos mamíferos. De fato, QUANDT \& GREENACRE (1999) obtiveram em seus estudos, tempo dilatado de indução de 200-338 segundos em psitacídeos. Esses autores utilizaram a CAM proposta para cães $(2,3 \mathrm{~V} \%)$, obtendo, desta forma, valores de indução maiores para o sevofluorano do que aqueles já descritos para o isoflurano. No que tange à recuperação da anestesia com o sevofluorano, esta ocorre em tempo bastante curto (4,26 minutos) e totalmente livre de excitações. O sevofluorano, cuja DAM é de 3,44\%, parece ser um anestésico bastante seguro para a espécie, pois não altera de forma deletéria as variáveis fisiológicas analisadas. Por isso pode ser considerado uma boa opção na prática anestésica em aves.

\section{REFERÊNCIAS BIBLIOGRÁFICAS}

ALTMAN, R.B. Twenty years of progress in avian anesthesia and surgery Journal of American Veterinary Medical Association, v.212, n.8, p.1233-1235, 1998.
BOEVER, W., WRIGHT, W. Use of ketamine for restrain \& anesthesia of birds Veterinary Medicine \& Small animal Clinician, v.70, n.1, p. 86-88, 1975.

BRAGA, A.F.A., BRAGA, F.S.S., POTÉRIO, G.M.B., et al Indução anestésica com sevofluorano em crianças. Alterações cardiocirculatórias e condições de intubação traqueal. Revista Brasileira de Anestesiologia, v.48, n.4, p.243-250, 1998.

CURRO, T.G., BRUNSON, D.B., PAUL-MURPHY, J. Determin ation of the ED50 of isoflurane and evaluation of the isoflurane-sparing effect of butorfhanol in cockatoos (cacatua spp.) Veterinary Surgery, n.23, p.429-433, 1994.

DOI, M., IKEDA, K. Airway irritation produced by volatile anesthetics during brief inhalation: comparasion of halothane, enflurane, isoflurane, and sevoflurane. Canadian Journal of Anesthesia, v.40, n.1, p.122-126, 1993.

EBERT, T.J., HARKIN, C.P., MUZI, M. Cardiovascualr responses to sevoflurane: a review. Anesthesia and Analgesia, v.81, p.S11-22, 1995.

FEDDE, M.R. Drugs used for avian anesthesia: a review. Poultry Science, v.57, n.5, p.1376-1399, 1978.

FRINK, E.J., MORGAN, S.E., COETZE, A., et al. The effects of sevoflurane, halothane, enflurane, and isoflurane on hepaticblood flow and oxigenation in chonically instrumented gryhound dogs. Anesthesiology, v.76, n.1, p.85-90, 1992.

GOELZ, M.F., HAHN, A.W., KELLEY, S.T. Effects of halothane and isoflurane on mean arterial blood pressure, heart rate, and respiratory rate in adult Pekin ducks. American Journal of Veterinary Ressearch, v.51, n.3,p.458-460, 1990.

GUIMARÃES, L.D., MORAES, A.N., CAMPELLO, R.A.V., $\boldsymbol{e}$ $a l$. Estudo comparativo entre sevofluorano, halotano e isoflurano em Gallus domesticus. Ciência Rural, v. 30, n.6, p.999-1004, 2000.

HALL, L.W., CLARKE, K.W. Anestesia veterinária. 8.ed. São Paulo : Manole, 1987. p.387-397.

HIKASA, Y., KAWANABE, H., TAKASE, K., $\boldsymbol{e}$ t $\boldsymbol{a}$. Comparisons of sevoflurane, isoflurane, and halothane anesthesia in spontaneiously breathing cats. Veterinary Anesthesia, v.25, n.2, p.234-243, 1996.

KORBEL, R. Vergleichende untersuchungen zur inhalationsanaästhesie mitisofluran (Forene $\left.{ }^{\circledR}\right)$ und sevofluran (SEVOrane ${ }^{\circledR}$ ) bei hausauben (Columba liviagmel.,1789, var. domestica) und vorstellung eines referenz - narkoseprotokolls für vögel. Tierärztl Prax, v.26, p.211-223, 1998.

LAURETTI, G.R., LIMA, I.C.P.R., MATTOS, A.L., et al. Indução inalatória gradativa com sevofluorano em pacientes adultas para cirurgias ginecológicas videolaparoscópicas. Revista Brasileira de Anestesiologia, v.48, n.2, p.119-125, 1998.

LUDDERS, J.W., MITCHELL, G.S., RODE, J. Minimmal anesthetic concentretion and cardiopulmonary dose response of isoflurane in ducks. Veterinary Surgery, v.19, n.4, p.304 307,1990

LUDDERS, J.W, MATTHEWS, N. Birds. In: LUMB, W.V.; JONES, E.W. Veterinary anesthesia. 3.ed. Baltimore: Willians \& Wilkins, 1996. Cap.20. p.645-669. 
LUMB, W.V., JONES, E.W. Veterinary anesthesia. 2.ed. Philadelphia: Lea \& Febiger, 1984. p.413-491

MÂNICA, J. Anestesiologia: princípi os e técnicas. 2.ed. Porto Alegre : Artes Médicas, 1997. Farmacodinâmica dos anestésicos inalatórios: p.251-270.

MUTOH, T., NISHIMURA, R., KIM, H. et al. Cardiopulmonary effects of sevoflurane, compared with halothane, enflurane, and isoflurane, in dogs. American Journal of Veterinary Ressearch, v.58, n.8, p.885-890, 1997.

NOCITI, J.R. Avaliação multicêntrica do sevofluorano. Revista Brasileira de Anestesiologia, v.48, n.1, p.29-36, 1998.

OLKOWSKY, A.A.; CLASSEN, H.L. Safety of isoflurane anaesthesia in high risk avian patients. Veterinary Record, v.143, n.3, p.82-83 1998

PAULIN, E. G. Respiratory pharmacology of inhalated anesthetic agents. In: MILLER, R.D. Anesthesia. 2.ed. New York : Chunchill Livingstone, 1986. p.667-699
QUANDT, J. E., GREENACRE, C.B. Sevoflurane anesthesia in psitaccines. Journal of Zoo and Wildlife Medicine, v.30, n.2, p.308-309,1999.

RITCHIE, B.W., HARRISON, G.J., HARRISON, L.R. Avian medicine: principles and apliccation. Florida: Wingers, 1997. 809p.

ROSSKOPF, W., WOERPEL, R. Diseases of cage and aviary birds 3.ed. Pennsylvania : Williams \& Wilkins, 1996. p.664-671

SCHMITT, P.M., GÖBEL, T., TRAUTVETTER, E. Evaluation of pulse oximetry as a monitoring method in avian anesthesia. Journal of Avian Medicine and Surgery, v.12, n.2, p.92-99, 1998.

STEFFEY, E. Inhalation anesthetics. In: THURMON, J.C., TRANQUILLY, W.J., BENSON, G.J. (eds.). Lumb's Jones veterinary anesthesia. 3.ed. Baltimore: Williams \& Wilkins, 1996. p.297-304

TERZI, R.G.G. Equilíbrio ácido-básico e transporte de oxigênio. São Paulo : Manole, 1992. 267p. 